\title{
Universiteit
}

Leiden

The Netherlands

\section{Collapse of optical vortices}

Vuong, L.T.; Grow, T.D.; Ishaaya, A.A.; Gaeta, A.L.; Hooft, G.W. 't; Eliel, E.R.

\section{Citation}

Vuong, L. T., Grow, T. D., Ishaaya, A. A., Gaeta, A. L., Hooft, G. W. 't, \& Eliel, E. R. (2006). Collapse of optical vortices. Physical Review Letters, 96(13), 133901.

doi:10.1103/PhysRevLett.96.133901

Version: $\quad$ Not Applicable (or Unknown)

License: $\quad$ Leiden University Non-exclusive license

Downloaded from: https://hdl.handle.net/1887/62711

Note: To cite this publication please use the final published version (if applicable). 


\title{
Collapse of Optical Vortices
}

\author{
Luat T. Vuong, Taylor D. Grow, Amiel Ishaaya, and Alexander L. Gaeta* \\ School of Applied and Engineering Physics, Cornell University, Ithaca, New York 14853, USA \\ Gert W. 't Hooft and Eric R. Eliel \\ Huygens Laboratory, Leiden University, P.O. Box 9504, 2300 RA Leiden, The Netherlands
}

Gadi Fibich

Department of Applied Mathematics, School of Mathematical Sciences, Tel Aviv University, Tel Aviv 69978, Israel

(Received 13 December 2005; published 6 April 2006)

\begin{abstract}
We theoretically and experimentally investigate the self-focusing of optical vortices in Kerr media. We observe collapse to a distinct self-similar profile, which becomes unstable to azimuthal perturbations. We analyze the azimuthal modulational instability for ring-shaped vortices and predict the number of azimuthal maxima solely as a function of power and topological charge. In our experiments, the observed multiple-filamentation patterns are in excellent agreement with our theoretical analysis.
\end{abstract}

DOI: 10.1103/PhysRevLett.96.133901

PACS numbers: 42.65.Sf

Pattern formation in nonlinear systems is investigated in a wide range of fields including optics, fluids, plasma physics, and material science [1]. In nonlinear optics, spatial features of an optical beam in a Kerr medium can arise from modulational instability (MI) [2], which can lead to multiple filamentation (MF), wherein each light filament holds a precise quanta of power and, in the absence of temporal effects or higher-order terms, will undergo collapse [3]. These MF patterns can be dramatically manipulated with beam shape [4,5], and the noise that seeds the MI does little to change the overall MF patterns [6].

Optical vortices with orbital angular momentum [7] reveal new dimensions of complexity in nonlinear beam propagation. These helical-phase beams have a strictly zero amplitude at the singularity where the phase is undefined and a topological charge $m$ that is a measure of the phase winding. Soliton vortex dynamics have been theoretically investigated in numerous contexts $[8,9]$, and the angular momentum and spatial dynamics have been studied experimentally in defocusing Kerr, photorefractive, quadratic, and saturable nonlinear media $[9,10]$. Some evidence of vortex MF has been observed [11]; however, these preliminary experiments were with powers below the critical threshold for vortex ring collapse.

In this Letter, we investigate the collapse of optical vortices in self-focusing Kerr media. In contrast to the MF of vortex rings in saturable media where an $m$-charge vortex tends to produce $2 m$ filaments [12], we find filamentation in a purely Kerr medium to be a function of both input power and $m$. We derive a new analytical relation that predicts the number of filaments which is in agreement with numerical simulations. Furthermore, we perform, to our knowledge, the first experimental study of collapsing vortices in Kerr media, the results of which are in excellent agreement with our predictions. The radially symmetric self-similar collapse of these beams, investigated previ- ously [8], is performed via a different formalism [13], and we show the agreement between simulation and analytical curves. Our analysis extends the previous work of vortex azimuthal MI $[14,15]$.

In our analysis, we use the nondimensionalized $(2+$ 1) $D$ nonlinear Schrödinger equation,

$$
\frac{\delta \psi}{\delta \zeta}-\frac{i}{4} \nabla_{\perp}^{2} \psi-i \frac{\alpha \pi P}{2 P_{\mathrm{cr}}}|\psi|^{2} \psi=0,
$$

where $\psi(\zeta, \rho, \theta)=A(\zeta, \rho, \theta) / \sqrt{\int|A(\zeta, \rho, \theta)|^{2} \rho d \rho d \theta}$, and $A$ is the electric field amplitude. The transformed coordinates are $\zeta=z / L_{\mathrm{df}}$ and $\rho=\sqrt{x^{2}+y^{2}} / r_{0}$, where $L_{\mathrm{df}}=$ $\frac{1}{2} k r_{0}^{2}$ is the diffraction length, $k=2 \pi n_{0} / \lambda$ is the wave number, $\lambda$ is the vacuum wavelength, $n_{0}$ is the linear index of refraction, and $r_{0}$ is the input radius of the mode field. Diffraction is described by the second term where the transverse Laplacian is given by $\nabla_{\perp}^{2}=\partial_{\rho, \rho}+\frac{1}{\rho} \partial_{\rho}+$ $\frac{1}{\rho^{2}} \partial_{\theta, \theta}$, and the third term is a result of the intensitydependent refractive index $n=n_{0}+n_{2} I$. It is characterized by the critical power for self-focusing $P_{\mathrm{cr}}=$ $\alpha\left(\lambda^{2} / 4 n_{0} n_{2}\right)$, where $\alpha$ is a constant dependent upon the initial beam shape [16]. The power is $P=\frac{n_{0} c}{2 \pi} \times$ $\int|A(\zeta, \rho, \theta)|^{2} r_{0} \rho r_{0} d \rho d \theta$.

A radially symmetric field with topological charge $m$ will approach a specific self-similar shape in a manner analogous to the evolution of $m=0$ beams to the Townes profile $[17,18]$. The critical power for vortex ring collapse for a Laguerre-Gaussian with vortex charge $m$ can be approximated as [8]

$$
P_{\mathrm{cr}}^{(m)}=\frac{2^{2 m+1} \Gamma(m+1) \Gamma(m+2)}{2 \Gamma(2 m+1)} P_{\mathrm{cr}} .
$$

Regardless of the initial shape, for powers above a critical threshold, the collapsing portion of the beam converges 
towards a distinct profile, in which the peak intensity scales inversely with the width, while the remaining portion diffracts. This self-similar profile $\psi_{\text {norm }}$ thus has the form $\psi(\zeta, \rho, \theta)_{\text {norm }}=L(\zeta) \psi\left(\zeta, \frac{\rho}{L(\zeta)}, \theta\right)$, where $L(\zeta)=\frac{1}{\max |\psi|}$. We define $\tilde{Y}_{m, P}$ to be the profile to which $\psi(\zeta, \rho, \theta)_{\text {norm }}$ is asymptotic as $\zeta$ tends to $\zeta_{c}$, which is the normalized distance at which $\psi$ becomes singular. To find $\tilde{Y}_{m, P}$ analytically, we use a general lens transformation for solutions of self-similar type near collapse in Eq. (1) [13], assume $L(\zeta) \sim \sigma \sqrt{\zeta_{c}-\zeta}$, and find that $\tilde{Y}_{m, P}$ obeys the equation

$$
\tilde{Y}^{\prime \prime}+\frac{1}{\tilde{\rho}} \tilde{Y}^{\prime}-\left[m^{2} / \tilde{\rho}^{2}-\sigma^{4} \tilde{\rho}^{2}+4\right] \tilde{Y}+\frac{2 \alpha \pi P}{P_{\mathrm{cr}}} \tilde{Y}^{3}=0,
$$

where $\tilde{\rho}=\frac{\rho}{L(\zeta)}$ is the normalized radius and $\tilde{Y}(\tilde{\rho}=0)=$ $\tilde{Y}(\tilde{\rho}=\infty)=0$. The Townes profile is equivalent to $\tilde{Y}_{m, P}$ with $m=0, \sigma=0$, and $P=P_{\mathrm{cr}}$. In Fig. 1 we plot $\tilde{Y}$ for different $m$ and $P$, determined by employing a shooting method, beginning close to zero and minimizing the power at a radius 10 times that of the peak field, fixing $\sigma$ for a solution with unit power, and finally renormalizing the result so that the peak field amplitude is 1 . Thus, $\tilde{Y}_{m, P}$ are the unique solutions of Eq. (3) defined by $m$ and $P$, with power and maximal norm of 1 .

In Fig. 2 we plot the evolution of a Laguerre-Gaussian input field with a topological charge of $m=1$ and $P=$ $4.4 P_{\mathrm{cr}} \approx 1.1 P_{\mathrm{cr}}^{(1)}$. After propagating $\zeta=0.5$, the intensity of $\psi_{\text {norm }}$ above the $1 / e^{2}$ point is nearly identical to the $\tilde{Y}$ profile. The ring intensity continues to increase and correspondingly shrink in both diameter and thickness as its normalized profile converges to $\tilde{Y}$. The relatively wide sampling of $[4096 \times 4096]$ points for $\rho=[-20,20]$ in the split-step numerical computation along with a superGaussian transmission window ensure that numerical noise and reflected power do not prematurely seed vortex breakup or cause vortex transmutation [19], and a nonuniform propagation stepsize minimizes simulation error. Similar convergence to $\tilde{Y}$ can be demonstrated with other radially symmetric input profiles of varying $m$ and $P$.

The addition of azimuthal noise alters collapse to a selfsimilar ring profile, and instead we observe breakup into a ring of individual filaments and investigate these MF pat-
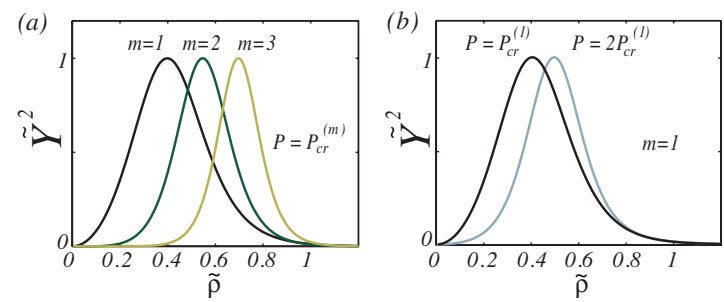

FIG. 1 (color online). Self-similar profiles $\tilde{Y}_{m, P}$ with (a) $P=P_{\mathrm{cr}}^{(m)}$ and $\sigma=0.47$ for $m=1,2, \quad$ and 3 (b) $P=4 P_{\mathrm{cr}} \approx P_{\mathrm{cr}}^{(1)}$ and $P=8 P_{\mathrm{cr}} \approx 2 P_{\mathrm{cr}}^{(1)}$, corresponding to $\sigma=0.47$ and $\sigma=0.38$, respectively, for $m=1$. terns. Because of the on-axis phase singularity where the field is strictly zero, optical vortex beams result in the formation of filaments off axis. For our perturbation analysis, instead of plane waves [14] or uniform-intensity rings [15], we use stationary field amplitudes resembling the Laguerre-Gaussian $\mathrm{TEM}_{p, l}$ modes, with the radial mode index $p=0$, the azimuthal index $l=m$, such that

$$
\begin{gathered}
\psi(\zeta, \rho, \theta)=C(1+\delta) \tilde{L G_{0, m}}(\rho, \theta), \\
\tilde{L G_{0, m}}(\rho, \theta)=\left(\frac{\rho}{w}\right)^{m} e^{-1 / 2(\rho / w)^{2}} e^{i m \theta},
\end{gathered}
$$

where $C$ and $w$ are the normalization terms for the electric field amplitude and mode field, respectively, as given by Eq. (1), and $\delta$ is the phase perturbation of azimuthal wave number $\eta$,

$$
\delta=\delta_{1}(\zeta) e^{i \eta \theta}+\delta_{2}(\zeta) e^{-i \eta \theta} .
$$

We assume the instability occurs at the radius of maximum intensity and substitute $\rho=\sqrt{m} w$. We look for solutions to $\delta_{1}(\zeta)$ and $\delta_{2}(\zeta)$ in the form of $e^{G \zeta}$, where $G$ has a nonvanishing real part, and identify the relation for $G$,

$$
G=\frac{\left(m+\frac{1}{2}\right)}{m} \sqrt{\tilde{P}_{m}^{2}-\left(\mu-2 \tilde{P}_{m}\right)^{2}}
$$

where $\tilde{P}_{m}=\frac{2 P \alpha m^{m}}{P_{\mathrm{cr}} e^{m}}$ and $\mu=2 m+m^{2}+\eta^{2}$. The wave numbers $\eta$ which satisfy Eq. (7) for positive $G$ will experience gain as plotted in Fig. 3(a). As the power increases, $G$ becomes wider and taller. As the power or topological charge increases, $G$ shifts towards higher $\eta$. It follows immediately from Eq. (7) that the maximum $G$ is attained at $\mu=2 \tilde{P}_{m}$ and is equal to $G_{\max }=2\left(m+\frac{1}{2}\right) P \alpha m^{m-1} / P_{\mathrm{cr}} e^{m}$ from which we can extrapolate that at higher powers,
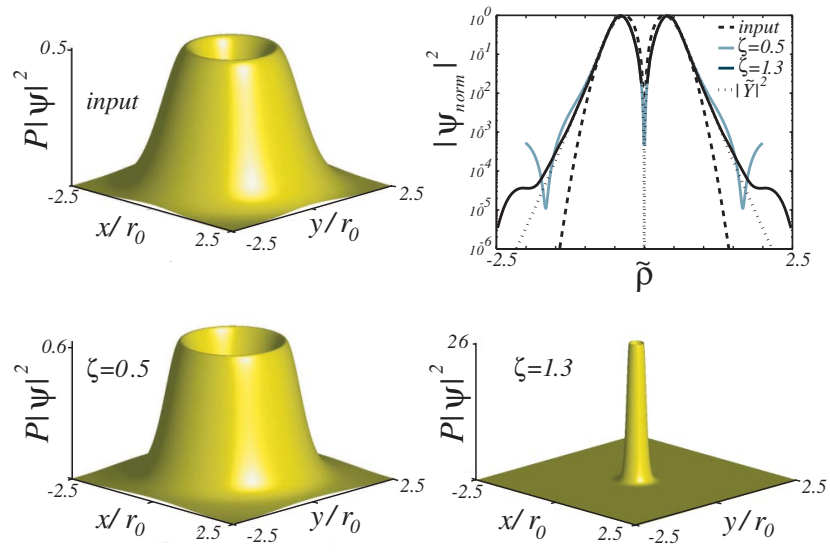

FIG. 2 (color online). Simulation plots for an input $\tilde{L G}_{0,1}$ with $P=4.4 P_{\mathrm{cr}} \approx 1.1 P_{\mathrm{cr}}^{(1)}$ at $\zeta=0,0.5$, and 1.3. Surface plots of $|\psi|^{2}$ are shown with the vertical axis scaled by $P$. Intensity cross sections of $\psi_{\text {norm }}$ are drawn for the input (dashed line), profiles at $\zeta=0.5$ and 1.3 (solid line), and the $\tilde{Y}$ to which $\psi_{\text {norm }}$ converges (dotted line). The intensity at $\tilde{\rho}=0$, which is equal to zero, is not shown. 

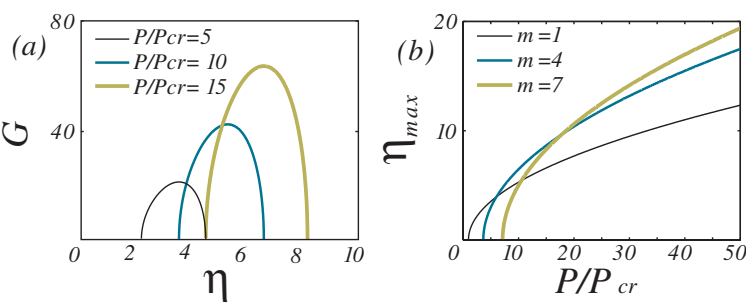

FIG. 3 (color online). (a) Gain $G$ of the azimuthal instability as a function of wave number $\eta$ for $m=1, P=5 P_{\mathrm{cr}} \approx 1.25 P_{\mathrm{cr}}^{(1)}$, $10 P_{\mathrm{cr}} \approx 2.5 P_{\mathrm{cr}}^{(1)}$, and $15 P_{\mathrm{cr}} \approx 3.75 P_{\mathrm{cr}}^{(1)}$. As the power increases, $G$ shifts to higher values of $\eta$. (b) Plot of the maximum-gain $\eta$ as a function of $P / P_{\mathrm{cr}}$ for $m=1,4$, and 7 .

vortices with higher topological charge are more susceptible to azimuthal MI. This relation for $G_{\max }$ yields the following prediction for the number of azimuthal maxima in the MF patterns for a given value of $P$ :

$$
\eta_{\max }=\sqrt{2 \tilde{P}_{m}-2 m-m^{2}} .
$$

Thus, we can approximate the wave number of maximal gain and the MF patterns solely as functions of beam power and topological charge. Plots for $\eta_{\max }$ as a function of $P$ for $m=1,4$, and 7 are shown in Fig. 3(b). We verified our model with the numerical code used to demonstrate selfsimilar collapse, and using $10 \%$ amplitude and phase noise, we find that our simulations match Eq. (8) for a topological charge up to at least $m=10$ (see Fig. 4). Our work is consistent with recent analysis [15], predicting $2 m+1$ azimuthal maxima at $P=P_{\mathrm{cr}}^{(m)}$. Moreover, our theory utilizes an integrable analytic field, the result of which is independent of ring radius and valid for powers other than $P_{\mathrm{cr}}^{(m)}$.

Above $P_{\mathrm{cr}}^{(m)}$, numerical simulations confirm the analytical prediction from Eq. (7) that higher-charge vortices are increasingly susceptible to azimuthal MI since MF occurs

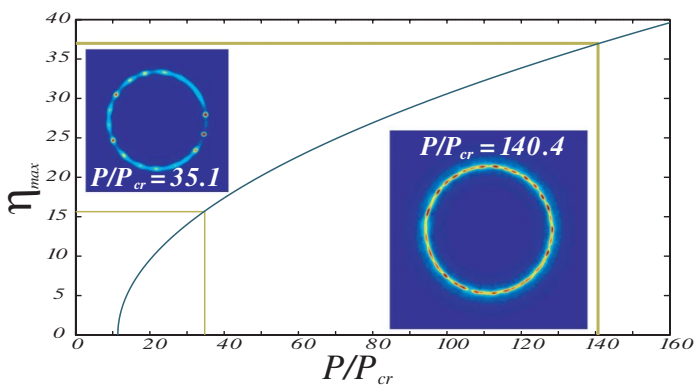

FIG. 4 (color online). Numerical and analytical results for topological charge $m=10$. The curve plots the wave number $\eta_{\max }$ of maximal gain as a function of $P / P_{\mathrm{cr}}$. The left inset shows the MF pattern of $P=35 P_{\mathrm{cr}} \approx 0.56 P_{\mathrm{cr}}^{(10)}$ at $\zeta=0.07$ from numerical simulations with 16 azimuthal maxima. The right inset shows that for $P=140 P_{\mathrm{cr}} \approx 2.24 P_{\mathrm{cr}}^{(10)}$ and 36 azimuthal maxima at $\zeta=0.02$. In both cases the analytical predictions are in excellent agreement with the simulation results. at shorter normalized distances for comparable noise and powers. At lower powers, spatial spreading results in higher stability to azimuthal MI [11]. We observe in our simulations that a vortex of $m=10$ and $P=20 P_{\mathrm{cr}} \approx$ $0.3 P_{\mathrm{cr}}^{(10)}$ with $5 \%$ noise neither forms azimuthal maxima nor collapses but instead diffracts as a ring. We find, however, the same $m=10$ vortex with $20 \%$ noise forms 10 filaments in agreement with analytical predictions. Our perturbation analysis utilizing a stationary profile accurately describes the MF of Laguerre-Gaussian beams for powers below $P_{\mathrm{cr}}^{(m)}$, and for powers above $P_{\mathrm{cr}}^{(m)}$, when breakup caused by MI is seeded initially. Under conditions where the vortex is more stable to MI and approaches the $\tilde{Y}$ profile before undergoing filamentation, our prediction for the number of filaments may become less accurate.

The experimental setup for investigating the collapse dynamics of optical vortices is shown in Fig. 5. Laguerre-Gaussian modes are produced using spiral phase plates with $90 \mathrm{fs}, 800 \mathrm{~nm}$ pulses of a Ti:Sapphire regenerative amplifier system. The phase plates are $m=1$ and are made from a polymer material using a high-precision molding technology [20]. Two plates are stacked to produce $m=2$ vortices. The telescoping is changed to vary the input beam width, and the final telescoping ranges from 50:1 to $2: 1$ demagnification. We estimate that the pulses have slightly broadened to $100 \mathrm{fs}$ after the telescoping. The vortices are propagated through an adjustable-length cell filled with water [21]. The time-averaged spatial beam profile from approximately 130 pulses is imaged with a 12-bit CCD camera (Spiricon LBA-FW-SCOR20). Distilled deionized water is used in the experiments, since it has a higher threshold for plasma formation than plain distilled water. Figure 5 shows the typical input intensity profile and its interference pattern with a flat-phase Gaussian beam. The latter shows a branch in the fringes verifying the $m=1$ and $m=2$ singularities produced by the phase plates.

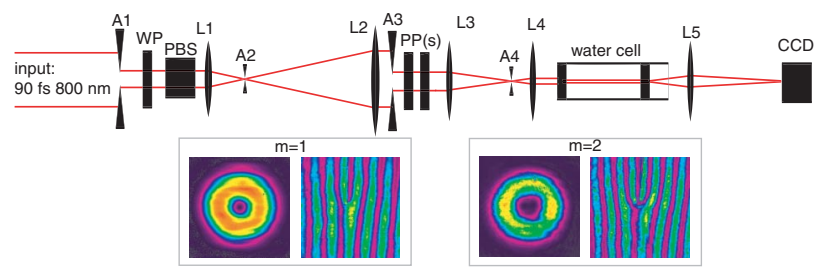

FIG. 5 (color online). Experimental setup. The half-wave plate WP and polarizing beam splitter PBS control the power. In the spatial filter L1-A2-L2, aperture A2 is a high-power tungsten pinhole. Aperture A3 selects a small portion of the Gaussian beam to transmit through the spiral phase plate(s) PP. Aperture A4 filters out higher-order Laguerre-Gaussian modes. The lower insets show typical transverse profiles incident upon the water cell and branching interference fringes demonstrating the vortex phase. The fringes are produced using a continuous-wave beam at $800 \mathrm{~nm}$ and by imaging the phase plate(s) with a Gaussian beam of zero topological charge. 

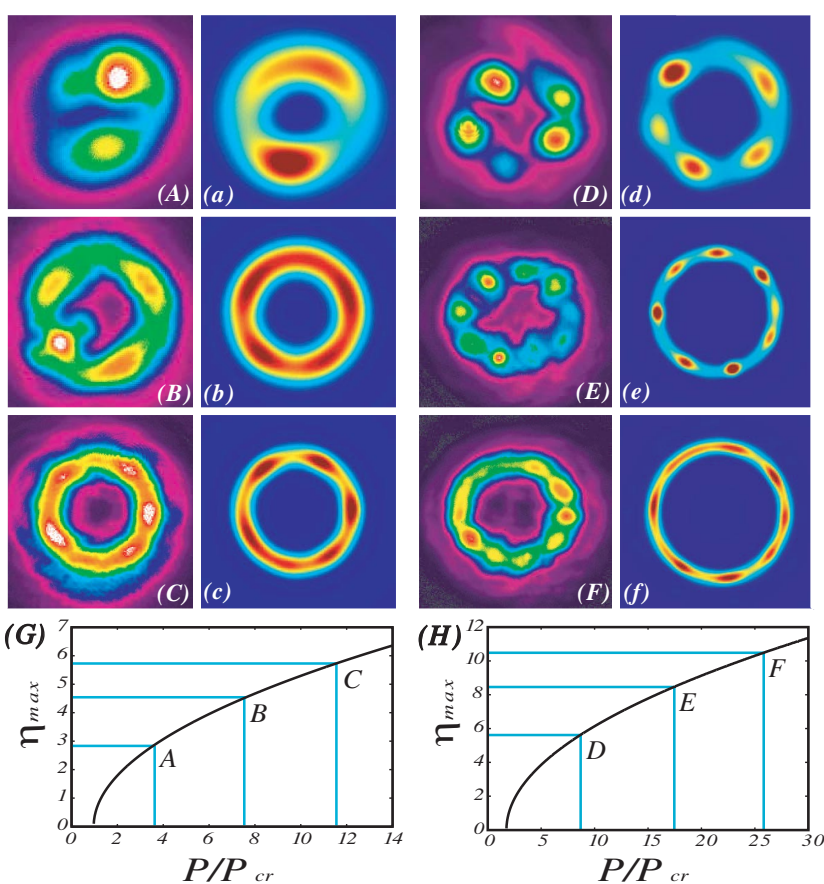

FIG. 6 (color online). Experimentally observed output profiles for $m=1$ with pulse energies (A) $6 \mu \mathrm{J}$, (B) $12 \mu \mathrm{J}$, (C) $18 \mu \mathrm{J}$, and corresponding simulations with powers (a) $P=3.85 P_{\mathrm{cr}} \approx$ $P_{\mathrm{cr}}^{(1)}$, (b) $P=7.7 P_{\mathrm{cr}} \approx 2 P_{\mathrm{cr}}^{(1)}$, (c) $P=11.6 P_{\mathrm{cr}} \approx 3 P_{\mathrm{cr}}^{(1)}$. Experimental pictures for $m=2$ with pulse energies (D) $6 \mu \mathrm{J}$, (E) $12 \mu \mathrm{J}$, (F) $18 \mu \mathrm{J}$, and corresponding simulations with powers (d) $P=8.6 P_{\mathrm{cr}} \approx 1.1 P_{\mathrm{cr}}^{(2)}$, (e) $P=17.2 P_{\mathrm{cr}} \approx 2.2 P_{\mathrm{cr}}^{(2)}$, (f) $P=$ $25.8 P_{\mathrm{cr}} \approx 3.3 P_{\mathrm{cr}}^{(2)}$. Plots of $\eta_{\max }$ vs $P / P_{\mathrm{cr}}$, with points along the curve marked to represent corresponding MF patterns shown for $m=1(\mathrm{G})$ and $m=2(\mathrm{H})$.

We propagate $m=1$ and $m=2$ vortices until just before the point at which supercontinuum generation is observed, which indicates that collapse has occurred [22], and modify the telescoping L3-L4 so that this distance to collapse is $15-20 \mathrm{~cm}$. Figs. 6(A), 6(a), 6(B), 6(b), 6(C), 6(c), 6(D), 6(d), 6(E), 6(e), 6(F), and 6(f) show experimental results and the corresponding simulations for $m=$ 1 and $m=2$, where doubling and tripling the power has the effect of changing the number of azimuthal maxima from 2 to 4 to 5 and from 5 to 8 to 10 , respectively. These results are highly repeatable and the number of azimuthal maxima, within the propagation distances described, vary at most by one. The CCD images are also highly stable, which we attribute to the pulse-to-pulse power stability of our laser system. The primary source of noise is inhomogeneity in the beam profile due to the alignment and imperfections of the PPs and the final aperture A4. Differences between experimental and simulation images are not surprising in view of these imperfections. However, while variations of the input profile affect the distance to collapse, the resulting $\eta$, within the lengths described, is virtually unchanged. We estimate that a $0.2 \mu \mathrm{J}, 100 \mathrm{fs}$ pulse has a peak power equal to one critical power. Our absolute power measurements are within a factor of 9 of our analytical predictions, and our relative power measurements are in excellent agreement as shown in Fig. 6(G) and $6(\mathrm{H})$. We believe that deviations in power from the theory are due to dispersive broadening effects that occur during propagation, which in our experiments is responsible for a decrease in peak power of $30 \%$.

In conclusion, we observe self-similar collapse of vortex beams. These beams are azimuthally unstable, and we derive an analytical expression for the number of azimuthal maxima in the breakup as a function of the power and topological charge, which predicts the MF of vortices both below and above the critical power for vortex collapse, $P_{\mathrm{cr}}^{(m)}$. Experimental results show this trend to be reliable for optical pulses undergoing collapse with powers as high as 3 $P_{\mathrm{cr}}^{(m)}$. Understanding MF behavior in the long-distance propagation of femtosecond laser pulses is valuable to remote-sensing applications [23]. Moreover, our results may also have relevance to vortices in Bose-Einstein condensates since the behavior of those systems has strong analogies with that of optical beams in Kerr media [9].

We gratefully acknowledge insightful discussions with S. Ghosh, funding from AT\&T Research Labs, the National Science Foundation under Grant No. PHY0244995, and the Army Research Office under Grant No. 48300-PH.

*Electronic address: a.gaeta@cornell.edu

[1] M. C. Cross and P. C. Hohenberg, Rev. Mod. Phys. 65, 851 (1993) and forward citations.

[2] V. I. Bespalov et al., JETP Lett. 3, 307 (1966).

[3] P. L. Kelley, Phys. Rev. Lett. 15, 1005 (1965).

[4] L. Bergé et al., Physica (Amsterdam) 176D, 181 (2003).

[5] A. Dubietis et al., Opt. Lett. 29, 1126 (2004).

[6] G. Fibich et al., Opt. Lett. 29, 1772 (2004).

[7] M. S. Soskin and M. V. Vasnetsov, Prog. Opt. 42, 219 (2001).

[8] V. I. Kruglov et al., J. Mod. Opt. 39, 2277 (1992).

[9] A. S. Desyatnikov et al., Prog. Opt. 47, 291 (2005).

[10] G. Duree et al., Phys. Rev. Lett. 74, 1978 (1995).

[11] M. Fisher et al., in Proceedings of the Conference for Lasers and Electro-Optics, Baltimore, Maryland, 2005, paper CMK7.

[12] M. S. Bigelow et al., Phys. Rev. Lett. 92, 083902 (2004).

[13] G. Fibich et al., Physica (Amsterdam) 211D, 193 (2005).

[14] J. M. Soto-Crespo et al., Phys. Rev. A 44, 636 (1991).

[15] A. Vinçotte and L. Bergé, Phys. Rev. Lett. 95, 193901 (2005).

[16] G. Fibich and A. L. Gaeta, Opt. Lett. 25, 335 (2000).

[17] R. Y. Chiao et al., Phys. Rev. Lett. 13, 479 (1964).

[18] K. D. Moll et al., Phys. Rev. Lett. 90, 203902 (2003).

[19] A. Ferrando et al., Phys. Rev. Lett. 95, 123901 (2005).

[20] S. Oemrawsingh et al., Appl. Opt. 43, 688 (2004).

[21] A. Dubietis et al., Phys. Rev. Lett. 92, 253903 (2004).

[22] A. L. Gaeta, Phys. Rev. Lett. 84, 3582 (2000).

[23] J. Kasparian et al., Science 301, 61 (2003). 